\title{
The $\Lambda_{b} \rightarrow \Lambda\left(\rightarrow p \pi^{-}\right) \mu^{+} \mu^{-}$decay in the aligned two-Higgs-doublet model
}

\author{
Quan-Yi Hu' ${ }^{\mathrm{a}}$, Xin-Qiang $\mathrm{Li}^{\mathrm{b}}$, Ya-Dong Yang ${ }^{\mathrm{c}}$ \\ Institute of Particle Physics and Key Laboratory of Quark and Lepton Physics (MOE), Central China Normal University, \\ Wuhan 430079, Hubei, China
}

Received: 29 January 2017 / Accepted: 27 March 2017 / Published online: 11 April 2017

(C) The Author(s) 2017. This article is an open access publication

\begin{abstract}
The rare baryonic decay $\Lambda_{b} \rightarrow \Lambda\left(\rightarrow p \pi^{-}\right)$ $\mu^{+} \mu^{-}$provides valuable complementary information compared to the corresponding mesonic $b \rightarrow s \mu^{+} \mu^{-}$transition. In this paper, using the latest high-precision lattice QCD calculation of the $\Lambda_{b} \rightarrow \Lambda$ transition form factors, we study this interesting decay within the aligned twoHiggs-doublet model, paying particularly attention to the effects of the chirality-flipped operators generated by the charged scalars. In order to extract the full set of angular coefficients in this decay, we consider the following ten angular observables, which can be derived from the analysis of the subsequent parity-violating $\Lambda \rightarrow p \pi^{-}$decay: the differential branching fraction $\mathrm{d} \mathcal{B} / \mathrm{d} q^{2}$, the longitudinal polarization fraction $F_{\mathrm{L}}$, the lepton-, hadron- and combined lepton-hadron-side forward-backward asymmetries $A_{\mathrm{FB}}^{\ell}, A_{\mathrm{FB}}^{\Lambda}$ and $A_{\mathrm{FB}}^{\ell \Lambda}$, as well as the other five asymmetry observables $Y_{i}(i=2,3 \mathrm{~s}, 3 \mathrm{sc}, 4 \mathrm{~s}, 4 \mathrm{sc})$. Detailed numerical comparisons are made between the SM and NP values for these angular observables. It is found that, under the constraints from the inclusive $B \rightarrow X_{s} \gamma$ branching fraction and

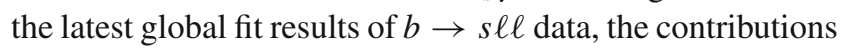
of right-handed semileptonic operators $O_{9,10}^{\prime}$, besides reconciling the $P_{5}^{\prime}$ anomaly observed in $B^{0} \rightarrow K^{* 0} \mu^{+} \mu^{-}$decay, could also enhance the values of $\mathrm{d} \mathcal{B} / \mathrm{d} q^{2}$ and $A_{\mathrm{FB}}^{\ell}$ in the bin $[15,20] \mathrm{GeV}^{2}$, leading to results consistent with the current $\mathrm{LHCb}$ measurements.
\end{abstract}

\section{Introduction}

The rare semileptonic $b$-hadron decays induced by the flavour-changing neutral current (FCNC) transition $b \rightarrow$ $s \ell^{+} \ell^{-}$do not arise at tree level and, due to the Glashow-

\footnotetext{
a e-mail: qyhu@mails.ccnu.edu.cn

be-mail: xqli@mail.ccnu.edu.cn

ce-mail: yangyd@mail.ccnu.edu.cn
}

Iliopoulos-Maiani (GIM) mechanism [1], are also highly suppressed at higher orders within the Standard Model (SM). In many extensions of the SM, on the other hand, new TeVscale particles can participate in the SM loop diagrams and lead to measurable effects in these rare decays. As a consequence, they play an important role in testing the SM and probing New Physics (NP) beyond it [2,3].

While no solid evidence of NP at all has been found in direct searches at high-energy colliders, it is interesting to note that several persistent deviations from the SM predictions have been observed in rare B-meson decays [3]. Specific to the $b \rightarrow s \ell^{+} \ell^{-}$mesonic decays, these include the angular observable $P_{5}^{\prime}$ in the kinematical distribution of $B^{0} \rightarrow K^{* 0} \mu^{+} \mu^{-}$[4-8], the lepton-flavour-universalityviolation ratio $R_{K}$ of the decay widths for $B \rightarrow K \mu^{+} \mu^{-}$and $B \rightarrow K e^{+} e^{-}$[9-11], as well as the differential decay rates for $B \rightarrow K^{(*)} \mu^{+} \mu^{-}$[12-14] and $B_{s} \rightarrow \phi \mu^{+} \mu^{-}$[15-17]. Motivated by these anomalies and using the other available data on such rare mesonic decays, several global analyses have been made [18-27], finding that a negative shift in the Wilson coefficient $C_{9}$ improves the agreement with the data. However, due to the large hadronic uncertainties involved in exclusive modes, it remains quite unclear whether these anomalies indicate the smoking gun of NP, or are caused merely by underestimated hadronic power corrections [2734] or even just by statistical fluctuations. In order to further understand the origin of the observed anomalies, it is very necessary to study other processes mediated by the same quark-level $b \rightarrow s \ell^{+} \ell^{-}$transition.

In this respect, the rare baryonic $\Lambda_{b} \rightarrow \Lambda \mu^{+} \mu^{-}$decay is of particular interest for the following two reasons. Firstly, due to the spin-half nature of $\Lambda_{b}$ and $\Lambda$ baryons, there is the potential to improve the currently limited understanding of the helicity structure of the underlying effective weak Hamiltonian [35-37]. Secondly, exploiting the full angular distribution of the four-body $\Lambda_{b} \rightarrow \Lambda\left(\rightarrow p \pi^{-}\right) \mu^{+} \mu^{-}$ 
decay, one can obtain information on the underlying shortdistance Wilson coefficients of effective four-fermion operators, which is complementary to that obtained from the corresponding mesonic decays [38-40]. Experimentally, this decay was observed firstly by the CDF collaboration with 24 signal events and a statistical significance of 5.8 Gaussian standard deviations [41]. Later, the LHCb collaboration published the first measurements of the differential branching fractions as well as three angular observables of this decay [42]. As the $\Lambda_{b}$ baryons account for around $20 \%$ of the $b$-hadrons produced at the LHC [43], refined measurements of this decay will be available in the near future. On the theoretical side, this decay is challenged by the hadronic uncertainties due to the $\Lambda_{b} \rightarrow \Lambda$ transition form factors and the non-factorizable spectator dynamics [38,44-46]. As the theory of QCD factorization at low $q^{2}[47,48]$ is not yet fully developed for the baryonic decay, we neglect all the non-factorizable spectator-scattering effects. For the factorizable nonlocal hadronic matrix elements of the operators $O_{1}-O_{6}, O_{8}$, we absorb them into the effective Wilson coefficients $C_{7}^{\text {eff }}\left(q^{2}\right)$ and $C_{9}^{\text {eff }}\left(q^{2}\right)$ [47-52]. For previous studies of this decay, the reader is referred to Refs. [53-80].

Interestingly, it has been observed by Meinel and Dyk [81] that the $\Lambda_{b} \rightarrow \Lambda\left(\rightarrow p \pi^{-}\right) \mu^{+} \mu^{-}$decay prefers a positive shift to the Wilson coefficient $C_{9}$, which is opposite in sign compared to that found in the latest global fits of only mesonic decays [22,26,27]. This suggests that a simple shift in $C_{9}$ alone could not explain all the current data and needs more thorough analyses. In our previous paper [82], we have studied the $B^{0} \rightarrow K^{* 0} \mu^{+} \mu^{-}$decay in the aligned two-Higgs-doublet model (A2HDM) [83], and found that the angular observable $P_{5}^{\prime}$ could be increased significantly to be consistent with the experimental data in the case when the charged-scalar contributions to $C_{7}^{\mathrm{H}^{ \pm}}$and $C_{9,10}^{\prime \mathrm{H}^{ \pm}}$are sizeable, but $C_{9,10}^{\mathrm{H}^{ \pm}} \simeq 0$. In order to further understand the anomalies observed in the $b \rightarrow s \ell^{+} \ell^{-}$mesonic decays, in this paper, we shall study the $\Lambda_{b} \rightarrow \Lambda\left(\rightarrow p \pi^{-}\right) \mu^{+} \mu^{-}$decay in the A2HDM. As the $\Lambda_{b}$ polarization in the LHCb setup has been measured to be small and compatible with zero [84], and the polarization effect will be averaged out for the symmetric ATLAS and CMS detectors, we consider only the case of unpolarized $\Lambda_{b}$ decay. In order to reduce as much as possible the uncertainties arising from input parameters and transition form factors, we shall calculate all of the angular observables in some appropriate combinations [38-40]. For the $\Lambda_{b} \rightarrow \Lambda$ transition form factors, we use the latest highprecision lattice QCD calculation [85], which is extrapolated to the whole $q^{2}$ region using the Bourrely-Caprini-Lellouch parametrization [86]. These results are also consistent with those of the recent QCD light-cone sum rule calculation [46], but with much smaller uncertainties in most of the kinematic range.
Our paper is organized as follows. In Sect. 2, we give a brief overview of the A2HDM. In Sect. 3, we present the theoretical framework for $\Lambda_{b} \rightarrow \Lambda\left(\rightarrow p \pi^{-}\right) \mu^{+} \mu^{-}$decay, including the effective weak Hamiltonian, the $\Lambda_{b} \rightarrow \Lambda$ transition form factors, and the observables of this decay. In Sect. 4, we give our numerical results and discussions. Our conclusions are made in Sect. 5. Some relevant formulae for the Wilson coefficients are collected in the appendix.

\section{The aligned two-Higgs-doublet model}

We consider the minimal version of $2 \mathrm{HDM}$, which is invariant under the SM gauge group and includes, besides the SM matter and gauge fields, two complex scalar $S U(2)_{L}$ doublets, with hypercharge $Y=1 / 2$ [83,87]. In the Higgs basis, the two doublets can be parametrized as

$\Phi_{1}=\left[\begin{array}{c}G^{+} \\ \frac{1}{\sqrt{2}}\left(v+S_{1}+i G^{0}\right)\end{array}\right], \quad \Phi_{2}=\left[\begin{array}{c}H^{+} \\ \frac{1}{\sqrt{2}}\left(S_{2}+i S_{3}\right)\end{array}\right]$,

where $v=\left(\sqrt{2} G_{F}\right)^{-1 / 2} \simeq 246 \mathrm{GeV}$ is the nonzero vacuum expectation value, and $G^{ \pm}, G^{0}$ are the massless Goldstone fields. The remaining five physical degrees of freedom are given by the two charged fields $H^{ \pm}(x)$ and the three neutral ones $\varphi_{i}^{0}(x)=\{h(x), H(x), A(x)\}=\mathcal{R}_{i j} S_{j}$, with the orthogonal transformation $\mathcal{R}$ fixed by the scalar potential $[83,87,88]$.

The most general Yukawa Lagrangian of the 2HDM is given by [83]

$$
\begin{aligned}
\mathcal{L}_{Y}= & -\frac{\sqrt{2}}{v}\left[\bar{Q}_{L}^{\prime}\left(M_{d}^{\prime} \Phi_{1}+Y_{d}^{\prime} \Phi_{2}\right) d_{R}^{\prime}+\bar{Q}_{L}^{\prime}\left(M_{u}^{\prime} \tilde{\Phi}_{1}\right.\right. \\
& \left.\left.+Y_{u}^{\prime} \tilde{\Phi}_{2}\right) u_{R}^{\prime}+\bar{L}_{L}^{\prime}\left(M_{\ell}^{\prime} \Phi_{1}+Y_{\ell}^{\prime} \Phi_{2}\right) \ell_{R}^{\prime}\right]+ \text { h.c. },
\end{aligned}
$$

where $\tilde{\Phi}_{a}(x) \equiv i \tau_{2} \Phi_{a}^{*}(x)$ are the charge-conjugated scalar doublets with hypercharge $Y=-\frac{1}{2} ; \bar{Q}_{L}^{\prime}$ and $\bar{L}_{L}^{\prime}$ are the lefthanded quark and lepton doublets, and $u_{R}^{\prime}, d_{R}^{\prime}$ and $\ell_{R}^{\prime}$ the corresponding right-handed singlets, in the weak-interaction basis. All fermionic fields are written as 3-dimensional vectors and the Yukawa couplings $M_{f}^{\prime}$ and $Y_{f}^{\prime}(f=u, d, \ell)$ are therefore $3 \times 3$ matrices in flavour space. Generally, the couplings $M_{f}^{\prime}$ and $Y_{f}^{\prime}$ cannot be diagonalized simultaneously and the non-diagonal elements will give rise to unwanted tree-level FCNC interactions. In the fermion mass-eigenstate basis, with diagonal mass matrices $M_{f}$, the tree-level FCNCs can be eliminated by requiring the alignment in flavour space of the Yukawa matrices [83]:

$Y_{d, \ell}=\varsigma_{d, \ell} M_{d, \ell}, \quad Y_{u}=\varsigma_{u}^{*} M_{u}$,

where $\varsigma_{f}(f=u, d, \ell)$ are arbitrary complex parameters and could introduce new sources of $\mathrm{CP}$ violation beyond the SM. 
The interactions of the charged scalars with the fermion mass-eigenstate fields read [83]

$$
\begin{aligned}
\mathcal{L}_{H^{ \pm}}= & -\frac{\sqrt{2}}{v} H^{+}\left\{\bar{u}\left[\varsigma_{d} V M_{d} P_{R}-\varsigma_{u} M_{u}^{\dagger} V P_{L}\right] d\right. \\
& \left.+\varsigma_{\ell} \bar{v} M_{\ell} P_{R} \ell\right\}+ \text { h.c. },
\end{aligned}
$$

where $P_{R(L)} \equiv \frac{1 \pm \gamma_{5}}{2}$ is the right (left)-handed chirality projector, and $V$ denotes the Cabibbo-Kobayashi-Maskawa (CKM) matrix [89,90]. As detailed in Refs. [82,88], the charged scalars could provide large contributions to $b \rightarrow$ $s \ell^{+} \ell^{-}$transitions, in some given parameter spaces.

\section{The $\Lambda_{b} \rightarrow \Lambda\left(\rightarrow p \pi^{-}\right) \mu^{+} \mu^{-}$decay}

\subsection{Effective weak Hamiltonian}

The effective weak Hamiltonian for $b \rightarrow s \ell^{+} \ell^{-}$transition is given by [52]

$$
\begin{aligned}
\mathcal{H}_{\mathrm{eff}}= & -\frac{4 G_{F}}{\sqrt{2}} V_{t b} V_{t s}^{*}\left[C_{1} O_{1}^{c}+C_{2} O_{2}^{c}\right. \\
& \left.+\sum_{i=3}^{10} C_{i} O_{i}+\sum_{i=7,9,10} C_{i}^{\prime} O_{i}^{\prime}\right],
\end{aligned}
$$

where $G_{F}$ is the Fermi coupling constant, and we have neglected the doubly Cabibbo-suppressed contributions to the decay amplitude. The operators $O_{i \leq 6}$ are identical to $P_{i}$ given in Ref. [91], and the remaining ones read

$O_{7}^{(\prime)}=\frac{e}{16 \pi^{2}} \bar{m}_{b}\left(\bar{s} \sigma^{\mu \nu} P_{R(L)} b\right) F_{\mu \nu}$,

$O_{8}=\frac{g_{s}}{16 \pi^{2}} \bar{m}_{b}\left(\bar{s} \sigma^{\mu \nu} T^{a} P_{R} b\right) G_{\mu \nu}^{a}$,

$O_{9}^{(\prime)}=\frac{e^{2}}{16 \pi^{2}}\left(\bar{s} \gamma^{\mu} P_{L(R)} b\right)\left(\bar{\ell} \gamma_{\mu} \ell\right)$

$O_{10}^{(\prime)}=\frac{e^{2}}{16 \pi^{2}}\left(\bar{s} \gamma^{\mu} P_{L(R)} b\right)\left(\bar{\ell} \gamma_{\mu} \gamma_{5} \ell\right)$,

where $e\left(g_{s}\right)$ is the electromagnetic (strong) coupling constant, $T^{a}$ the generator of $S U(3)_{C}$ in the fundamental representation, and $\bar{m}_{b}$ denotes the $b$-quark running mass in the $\overline{\mathrm{MS}}$ scheme.

Within the SM, $O_{7,9,10}$ play the leading role in $b \rightarrow$ $s \ell^{+} \ell^{-}$transition, while the factorizable contributions from $O_{1-6,8}$ can be absorbed into the effective Wilson coefficients $C_{7}^{\text {eff }}\left(q^{2}\right)$ and $C_{9}^{\text {eff }}\left(q^{2}\right)[25]$ :

$$
\begin{aligned}
C_{7}^{\mathrm{eff}}\left(q^{2}\right)= & C_{7}-\frac{1}{3}\left(C_{3}+\frac{4}{3} C_{4}+20 C_{5}+\frac{80}{3} C_{6}\right) \\
& -\frac{\alpha_{s}}{4 \pi}\left[\left(C_{1}-6 C_{2}\right) F_{1, c}^{(7)}\left(q^{2}\right)+C_{8} F_{8}^{(7)}\left(q^{2}\right)\right],
\end{aligned}
$$

$$
\begin{aligned}
C_{9}^{\mathrm{eff}}\left(q^{2}\right)= & C_{9}+\frac{4}{3}\left(C_{3}+\frac{16}{3} C_{5}+\frac{16}{9} C_{6}\right) \\
& -h\left(0, q^{2}\right)\left(\frac{1}{2} C_{3}+\frac{2}{3} C_{4}+8 C_{5}+\frac{32}{3} C_{6}\right) \\
& -h\left(m_{b}, q^{2}\right)\left(\frac{7}{2} C_{3}+\frac{2}{3} C_{4}+38 C_{5}+\frac{32}{3} C_{6}\right) \\
& +h\left(m_{c}, q^{2}\right)\left(\frac{4}{3} C_{1}+C_{2}+6 C_{3}+60 C_{5}\right) \\
& -\frac{\alpha_{s}}{4 \pi}\left[C_{1} F_{1, c}^{(9)}\left(q^{2}\right)+C_{2} F_{2, c}^{(9)}\left(q^{2}\right)+C_{8} F_{8}^{(9)}\left(q^{2}\right)\right],
\end{aligned}
$$

where the basic fermion loop function is given by [47]

$$
\begin{aligned}
& h\left(m_{q}, q^{2}\right)=\frac{4}{9}\left(\ln \frac{\mu^{2}}{m_{q}^{2}}+\frac{2}{3}+z\right) \\
& -\frac{4}{9}(2+z) \sqrt{|z-1|} \begin{cases}\arctan \frac{1}{\sqrt{z-1}}, & z=\frac{4 m_{q}^{2}}{q^{2}}>1, \\
\ln \frac{1+\sqrt{1-z}}{\sqrt{z}}-\frac{i \pi}{2}, & z=\frac{4 m_{q}^{2}}{q^{2}} \leq 1,\end{cases}
\end{aligned}
$$

and the functions $F_{8}^{(7,9)}\left(q^{2}\right)$ are defined by Eqs. (B.1) and (B.2) of Ref. [47], while $F_{1, c}^{(7,9)}\left(q^{2}\right)$ and $F_{2, c}^{(7,9)}\left(q^{2}\right)$ are provided in Ref. [92] for low $q^{2}$ and in Ref. [93] for high $q^{2}$. The quark masses appearing in these functions are defined in the pole scheme. The contribution from $O_{7}^{\prime}$ is suppressed by $\bar{m}_{s} / \bar{m}_{b}$ and those from $O_{9,10}^{\prime}$ are zero within the SM.

In the A2HDM, the charged-scalar exchanges lead to additional contributions to $C_{7,9,10}$ and make the contributions of chirality-flipped operators $O_{7,9,10}^{\prime}$ to be significant, through the $Z^{0}$ - and photon-penguin diagrams shown in Fig. 1. Since we have neglected the light lepton mass, there is no contribution from the SM $W$-box diagrams with the $W^{ \pm}$bosons replaced by the charged scalars $H^{ \pm}$. The new contributions to the Wilson coefficients read [82]

$$
\begin{aligned}
C_{7}^{\mathrm{H}^{ \pm}} & =\left|\varsigma_{u}\right|^{2} C_{7, \mathrm{uu}}+\varsigma_{d} \varsigma_{u}^{*} C_{7, \mathrm{ud}}, \\
C_{9}^{\mathrm{H}^{ \pm}} & =\left|\varsigma_{u}\right|^{2} C_{9, \mathrm{uu}}, \\
C_{10}^{\mathrm{H}^{ \pm}} & =\left|\varsigma_{u}\right|^{2} C_{10, \mathrm{uu}}, \\
C_{7}^{\prime \mathrm{H}^{ \pm}} & =\frac{\bar{m}_{s}}{\bar{m}_{b}}\left[\left|\varsigma_{u}\right|^{2} C_{7, \mathrm{uu}}+\varsigma_{u} \varsigma_{d}^{*} C_{7, \mathrm{ud}}\right],
\end{aligned}
$$

\footnotetext{
${ }^{1}$ Here we incorporate only the leading contributions from an operator product expansion (OPE) of the nonlocal product of $O_{1-6,8}$ with the quark electromagnetic current, because the first and second-order corrections in $\Lambda / m_{b}$ from the OPE are already well suppressed in the high- $q^{2}$ region $[49,50]$. Although non-factorizable spectator-scattering effects (i.e., corrections that are not described using hadronic form factors) are expected to play a sizeable role in the low- $q^{2}$ region $[47,48]$, we shall neglect their contributions because there is presently no systematic framework in which they can be calculated for the baryonic decay [46]. As a consequence, our predictions in the low- $q^{2}$ region are affected by a hitherto unquantified systematic uncertainty.
} 


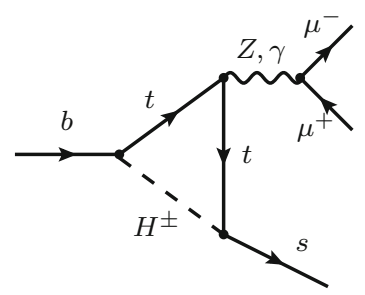

$(1.1)$

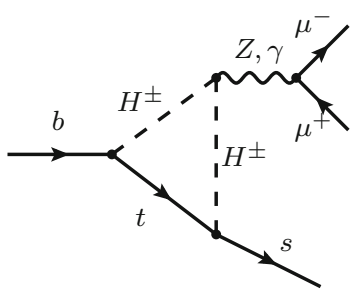

$(1.2)$

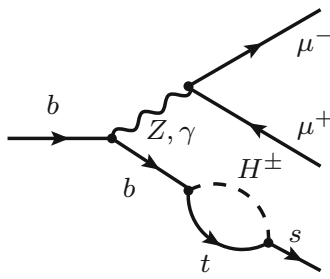

(1.3)

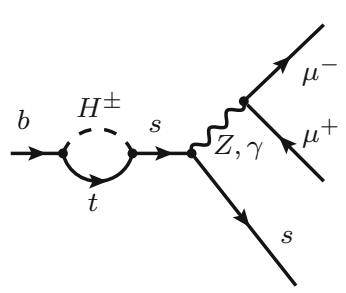

(1.4)

Fig. $1 Z$ - and photon-penguin diagrams involving the charged-scalar exchanges in the A2HDM

$$
\begin{aligned}
C_{9}^{\prime \mathrm{H}^{ \pm}}= & \left(-1+4 \sin ^{2} \theta_{W}\right) C_{10}^{\prime \mathrm{H}^{ \pm}} \\
& +\frac{\bar{m}_{b} \bar{m}_{s}}{m_{W}^{2}}\left[\left|\varsigma_{u}\right|^{2} C_{9, \mathrm{uu}}^{\prime}+2 \Re\left(\varsigma_{u} \varsigma_{d}^{*}\right) C_{9, \mathrm{ud}}^{\prime}\right. \\
& \left.+\left|\varsigma_{d}\right|^{2} C_{9, \mathrm{dd}}^{\prime}\right], \\
C_{10}^{\prime \mathrm{H}^{ \pm}}= & \frac{\bar{m}_{b} \bar{m}_{s}}{m_{W}^{2}}\left[\left|\varsigma_{u}\right|^{2} C_{10, \mathrm{uu}}^{\prime}+2 \Re\left(\varsigma_{u} \varsigma_{d}^{*}\right) C_{10, \mathrm{ud}}^{\prime}\right. \\
& \left.+\left|\varsigma_{d}\right|^{2} C_{10, \mathrm{dd}}^{\prime}\right],
\end{aligned}
$$

with the functions $C_{i, \mathrm{XY}}^{(\prime)}(i=7,9,10 ; \mathrm{X}, \mathrm{Y}=\mathrm{u}, \mathrm{d})$ given by Eqs. (A.1)-(A.10). Assuming $\varsigma_{u, d}$ to be real, one has $C_{7}^{\prime \mathrm{H}^{ \pm}}=\frac{\bar{m}_{s}}{\bar{m}_{b}} C_{7}^{\mathrm{H}^{ \pm}}$, and we shall therefore neglect $C_{7}^{\prime \mathrm{H}^{ \pm}}$in the following discussion.

\subsection{Transition form factors}

In order to obtain compact forms of the helicity amplitudes [38], we adopt the helicity-based definitions of the $\Lambda_{b} \rightarrow \Lambda$ transition form factors, which are given by $[38,44]$

$$
\begin{aligned}
\langle\Lambda & \left.\left(p^{\prime}, s^{\prime}\right)\left|\bar{s} \gamma^{\mu} b\right| \Lambda_{b}(p, s)\right\rangle \\
= & \bar{u}_{\Lambda}\left(p^{\prime}, s^{\prime}\right)\left[f_{t}^{V}\left(q^{2}\right)\left(m_{\Lambda_{b}}-m_{\Lambda}\right) \frac{q^{\mu}}{q^{2}}+f_{0}^{V}\left(q^{2}\right)\right. \\
& \times \frac{m_{\Lambda_{b}}+m_{\Lambda}}{s_{+}}\left(p^{\mu}+p^{\prime \mu}-\left(m_{\Lambda_{b}}^{2}-m_{\Lambda}^{2}\right) \frac{q^{\mu}}{q^{2}}\right) \\
& \left.+f_{\perp}^{V}\left(q^{2}\right)\left(\gamma^{\mu}-\frac{2 m_{\Lambda}}{s_{+}} p^{\mu}-\frac{2 m_{\Lambda_{b}}}{s_{+}} p^{\prime \mu}\right)\right] u_{\Lambda_{b}}(p, s),
\end{aligned}
$$

$$
\begin{aligned}
& \left\langle\Lambda\left(p^{\prime}, s^{\prime}\right)\left|\bar{s} \gamma^{\mu} \gamma_{5} b\right| \Lambda_{b}(p, s)\right\rangle \\
& \quad=-\bar{u}_{\Lambda}\left(p^{\prime}, s^{\prime}\right) \gamma_{5}\left[f_{t}^{A}\left(q^{2}\right)\left(m_{\Lambda_{b}}+m_{\Lambda}\right) \frac{q^{\mu}}{q^{2}}\right. \\
& \quad+f_{0}^{A}\left(q^{2}\right) \frac{m_{\Lambda_{b}}-m_{\Lambda}}{s_{-}}\left(p^{\mu}+p^{\prime \mu}-\left(m_{\Lambda_{b}}^{2}-m_{\Lambda}^{2}\right) \frac{q^{\mu}}{q^{2}}\right) \\
& \left.\quad+f_{\perp}^{A}\left(q^{2}\right)\left(\gamma^{\mu}+\frac{2 m_{\Lambda}}{s_{-}} p^{\mu}-\frac{2 m_{\Lambda_{b}}}{s_{-}} p^{\prime \mu}\right)\right] u_{\Lambda_{b}}(p, s),
\end{aligned}
$$

for the vector and axial-vector currents, respectively, and

$$
\begin{aligned}
& \left\langle\Lambda\left(p^{\prime}, s^{\prime}\right)\left|\bar{s} i \sigma^{\mu v} q_{\nu} b\right| \Lambda_{b}(p, s)\right\rangle \\
& =-\bar{u}_{\Lambda}\left(p^{\prime}, s^{\prime}\right)\left[f_{0}^{T}\left(q^{2}\right) \frac{q^{2}}{s_{+}}\left(p^{\mu}+p^{\prime \mu}-\left(m_{\Lambda_{b}}^{2}-m_{\Lambda}^{2}\right) \frac{q^{\mu}}{q^{2}}\right)\right. \\
& \left.\quad+f_{\perp}^{T}\left(q^{2}\right)\left(m_{\Lambda_{b}}+m_{\Lambda}\right)\left(\gamma^{\mu}-\frac{2 m_{\Lambda}}{s_{+}} p^{\mu}-\frac{2 m_{\Lambda_{b}}}{s_{+}} p^{\prime \mu}\right)\right] u_{\Lambda_{b}}(p, s),
\end{aligned}
$$

$$
\begin{aligned}
& \left\langle\Lambda\left(p^{\prime}, s^{\prime}\right)\left|\bar{s} i \sigma^{\mu v} \gamma_{5} q_{v} b\right| \Lambda_{b}(p, s)\right\rangle \\
& =-\bar{u}_{\Lambda}\left(p^{\prime}, s^{\prime}\right) \gamma_{5}\left[f_{0}^{T_{5}}\left(q^{2}\right) \frac{q^{2}}{s_{-}}\left(p^{\mu}+p^{\prime \mu}-\left(m_{\Lambda_{b}}^{2}-m_{\Lambda}^{2}\right) \frac{q^{\mu}}{q^{2}}\right)\right. \\
& \left.\quad+f_{\perp}^{T_{5}}\left(q^{2}\right)\left(m_{\Lambda_{b}}-m_{\Lambda}\right)\left(\gamma^{\mu}+\frac{2 m_{\Lambda}}{s_{-}} p^{\mu}-\frac{2 m_{\Lambda_{b}}}{s_{-}} p^{\prime \mu}\right)\right] u_{\Lambda_{b}}(p, s),
\end{aligned}
$$

for the tensor and pseudo-tensor currents, respectively. Here $q=p-p^{\prime}$ and $s_{ \pm}=\left(m_{\Lambda_{b}} \pm m_{\Lambda}\right)^{2}-q^{2}$. The helicity form factors satisfy the endpoint relations $f_{t}^{V(A)}(0)=f_{0}^{V(A)}(0)$ and $f_{\perp}^{A\left(T_{5}\right)}\left(q_{\max }^{2}\right)=f_{0}^{A\left(T_{5}\right)}\left(q_{\max }^{2}\right)$, with $q_{\max }^{2}=\left(m_{\Lambda_{b}}-\right.$ $\left.m_{\Lambda}\right)^{2}$. All these ten form factors have been recently calculated using $(2+1)$-flavour lattice QCD [85].

\subsection{Observables in $\Lambda_{b} \rightarrow \Lambda\left(\rightarrow p \pi^{-}\right) \mu^{+} \mu^{-}$decay}

The angular distribution of the four-body $\Lambda_{b} \rightarrow \Lambda(\rightarrow$ $\left.p \pi^{-}\right) \mu^{+} \mu^{-}$decay, with an unpolarized $\Lambda_{b}$, is described by the dimuon invariant mass squared $q^{2}$, the helicity angles $\theta_{\Lambda}$ and $\theta_{\ell}$, and the azimuthal angle $\phi$; the explicit definition of these four kinematic variables could be found, for example, in Refs. [38,39]. The four-fold differential width can then be written as [38]

$$
\begin{aligned}
\frac{\mathrm{d}^{4} \Gamma}{\mathrm{d} q^{2} \mathrm{~d} \cos \theta_{\ell} \mathrm{d} \cos \theta_{\Lambda} \mathrm{d} \phi}= & \frac{3}{8 \pi}\left[\left(K_{1 s s} \sin ^{2} \theta_{\ell}+K_{1 c c} \cos ^{2} \theta_{\ell}\right.\right. \\
& \left.+K_{1 c} \cos \theta_{\ell}\right) \\
& +\left(K_{2 s s} \sin ^{2} \theta_{\ell}+K_{2 c c} \cos ^{2} \theta_{\ell}\right. \\
& \left.+K_{2 c} \cos \theta_{\ell}\right) \cos \theta_{\Lambda} \\
& +\left(K_{3 s c} \sin \theta_{\ell} \cos \theta_{\ell}\right. \\
& \left.+K_{3 s} \sin \theta_{\ell}\right) \sin \theta_{\Lambda} \cos \phi
\end{aligned}
$$




$$
\begin{aligned}
& +\left(K_{4 s c} \sin \theta_{\ell} \cos \theta_{\ell}\right. \\
& \left.\left.+K_{4 s} \sin \theta_{\ell}\right) \sin \theta_{\Lambda} \sin \phi\right],
\end{aligned}
$$

where the angular coefficients $K_{n \lambda}$, with $n=1, \ldots, 4$ and $\lambda=s, c, s s, c c, s c$, are functions of $q^{2}$, and can be expressed in terms of eight transversity amplitudes for $\Lambda_{b} \rightarrow \Lambda$ transition and the parity-violating decay parameter $\alpha_{\Lambda}$ in the secondary decay $\Lambda \rightarrow p \pi^{-}$; their explicit expressions could be found in Ref. [38].

Starting with Eq. (3.17) and in terms of the angular coefficients $K_{n \lambda}$, we can then construct the following observables [38-40]:

- The differential decay rate and differential branching fraction

$$
\frac{\mathrm{d} \Gamma}{\mathrm{d} q^{2}}=2 K_{1 s s}+K_{1 c c}, \quad \frac{\mathrm{d} \mathcal{B}}{\mathrm{d} q^{2}}=\tau_{\Lambda_{b}} \frac{\mathrm{d} \Gamma}{\mathrm{d} q^{2}},
$$

where $\tau_{\Lambda_{b}}$ is the $\Lambda_{b}$ lifetime.

- The longitudinal polarization fraction of the dimuon system

$$
F_{\mathrm{L}}=2 \hat{K}_{1 s s}-\hat{K}_{1 c c},
$$

where we introduce the normalized angular observables $\hat{K}_{n \lambda}=\frac{K_{n \lambda}}{\mathrm{d} \Gamma / \mathrm{d} q^{2}}$.

- The lepton-, hadron- and combined lepton-hadron-side forward-backward asymmetries

$$
A_{\mathrm{FB}}^{\ell}=\frac{3}{2} \hat{K}_{1 c}, \quad A_{\mathrm{FB}}^{\Lambda}=\hat{K}_{2 s s}+\frac{1}{2} \hat{K}_{2 c c}, \quad A_{\mathrm{FB}}^{\ell \Lambda}=\frac{3}{4} \hat{K}_{2 c},
$$

which have characteristic $q^{2}$ behaviours: within the SM, both $A_{\mathrm{FB}}^{\ell}$ and $A_{\mathrm{FB}}^{\ell \Lambda}$ have the same zero-crossing points, $q_{0}^{2}\left(A_{\mathrm{FB}}^{\ell}\right) \simeq q_{0}^{2}\left(A_{\mathrm{FB}}^{\ell \Lambda}\right)$, to the first approximation, while $A_{\mathrm{FB}}^{\Lambda}$ does not cross zero [38]. Note that, as observed in the mesonic case [47,94-96], the zero-crossing points are nearly free of hadronic uncertainties [38-40].

- The other five asymmetry observables

$$
\begin{aligned}
& Y_{2}=\frac{3}{8}\left(\hat{K}_{2 c c}-\hat{K}_{2 s s}\right), \quad Y_{3 \mathrm{~s}}=\frac{3 \pi}{8} \hat{K}_{3 s}, \quad Y_{3 \mathrm{sc}}=\frac{1}{2} \hat{K}_{3 s c} \\
& Y_{4 \mathrm{~s}}=\frac{3 \pi}{8} \hat{K}_{4 s}, \quad Y_{4 \mathrm{sc}}=\frac{1}{2} \hat{K}_{4 s c}
\end{aligned}
$$

which, along with the previous observables, determine all the ten angular coefficients $K_{n \lambda}$. Here $Y_{2}$ also has a zero-crossing point, which lies in the low $q^{2}$ region.

In order to compare with the experimental data [97], we also consider the binned differential branching fraction defined by $\left\langle\mathrm{d} \mathcal{B} / \mathrm{d} q^{2}\right\rangle_{\left[q_{\min }^{2}, q_{\max }^{2}\right]}=\frac{\int_{q_{\min }^{2}}^{q_{\max }^{2}}\left(\mathrm{~d} \mathcal{B} / \mathrm{d} q^{2}\right) \mathrm{d} q^{2}}{q_{\max }^{2}-q_{\min }^{2}}$,

and the binned normalized angular coefficients defined by

$\left\langle\hat{K}_{n \lambda}\right\rangle_{\left[q_{\min }^{2}, q_{\max }^{2}\right]}=\frac{\int_{q_{\min }^{2}}^{q_{\max }^{2}} K_{n \lambda} \mathrm{d} q^{2}}{\int_{q_{\min }^{2}}^{q_{\max }^{2}}\left(\mathrm{~d} \Gamma / \mathrm{d} q^{2}\right) \mathrm{d} q^{2}}$,

where the numerator and denominator should be binned separately. As the theoretical calculations are thought to break down close to the narrow charmonium resonances, we make no predictions for these observables in this region.

Finally, it should be noted that, unlike the strong decay $K^{*} \rightarrow K \pi$ in the mesonic counterpart $B \rightarrow K^{*} \ell^{+} \ell^{-}$, the subsequent weak decay $\Lambda \rightarrow p \pi^{-}$is parity violating, with the asymmetry parameter $\alpha_{\Lambda}$ being known from experiment [98]. This fact distinguishes the signal with an intermediate $\Lambda$ baryon from the direct $\Lambda_{b} \rightarrow p \pi^{-} \mu^{+} \mu^{-}$ decay, and facilitates the full angular analysis of $\Lambda_{b} \rightarrow \Lambda(\rightarrow$ $\left.p \pi^{-}\right) \mu^{+} \mu^{-}$decay $[38,39]$.

\section{Numerical results and discussions}

\subsection{Input parameters}

Firstly we collect in Table 1 the theoretical input parameters entering our numerical analysis throughout this paper. These include the SM parameters such as the electromagnetic and strong coupling constants, gauge boson, quark and hadron masses, ${ }^{2}$ as well as the CKM matrix elements. The Weinberg mixing angle $\theta_{W}$ is given by $\sin ^{2} \theta_{W}=1-M_{W}^{2} / M_{Z}^{2}$.

For the $\Lambda_{b} \rightarrow \Lambda$ transition form factors, we use the latest high-precision lattice QCD calculation with $2+1$ dynamical flavours [85]. The $q^{2}$ dependence of these form factors are parametrized in a simplified $z$ expansion [86], modified to account for pion-mass and lattice-spacing dependences. All relevant formulae and input parameters can be found in Eqs. (38) and (49) and Tables III-V and IX-XII of Ref. [85]. To compute the central value, statistical uncertainty, and total systematic uncertainty of any observable depending on the form factors, such as the differential branching fraction and angular observables given in Eqs. (3.18)-(3.21), as well as the corresponding binned observables and the zerocrossing points, we follow the same procedure as specified in Eqs. (50)-(55) of Ref. [85].

2 The pion mass is needed to describe the secondary decay $\Lambda \rightarrow p \pi^{-}$, and the kaon and $B$-meson masses are used to evaluate the $B K$ threshold in the $z$ parametrization of the $\Lambda_{b} \rightarrow \Lambda$ transition form factors [85]. 
Table 1 Summary of the theoretical input parameters used throughout this paper

\begin{tabular}{|c|c|c|c|c|c|c|}
\hline \multicolumn{7}{|c|}{ QCD and electroweak parameters } \\
\hline$G_{F}\left[10^{-5} \mathrm{GeV}^{-2}\right]$ & $\alpha_{s}\left(m_{Z}\right)$ & $\alpha_{e}\left(m_{W}\right)$ & $m_{Z}(\mathrm{GeV})$ & $m_{W}(\mathrm{GeV})$ & & \multirow[t]{2}{*}{ [98] } \\
\hline 1.1663787 & $0.1182 \pm 0.0012$ & $1 / 128$ & 91.1876 & 80.385 & & \\
\hline \multicolumn{7}{|l|}{ Quark masses (GeV) } \\
\hline$m_{t}^{\text {pole }}$ & $m_{b}^{\text {pole }}$ & $m_{c}^{\text {pole }}$ & $\bar{m}_{b}\left(\bar{m}_{b}\right)$ & $\bar{m}_{c}\left(\bar{m}_{c}\right)$ & $\bar{m}_{s}(2 \mathrm{GeV})$ & \multirow[t]{2}{*}{ [98] } \\
\hline $174.2 \pm 1.4$ & $4.78 \pm 0.06$ & $1.67 \pm 0.07$ & $4.18 \pm 0.03$ & $1.27 \pm 0.03$ & $0.096_{-0.004}^{+0.008}$ & \\
\hline \multicolumn{7}{|c|}{ Meson and baryon masses $(\mathrm{GeV})$} \\
\hline$m_{\pi}$ & $m_{K}$ & $m_{B}$ & $m_{\Lambda}$ & $m_{\Lambda_{b}}$ & & \multirow[t]{2}{*}[85,98]{} \\
\hline 0.135 & 0.494 & 5.279 & 1.116 & 5.619 & & \\
\hline \multicolumn{7}{|l|}{ Other parameters } \\
\hline$\Lambda_{b}$ lifetime $\left(\tau_{\Lambda_{b}}\right)$ & & Parity-violating parameter $\left(\alpha_{\Lambda}\right)$ & & $\left|V_{t s}^{*} V_{t b}\right|$ & & \multirow[t]{2}{*}[98,99]{} \\
\hline$(1.466 \pm 0.010) \mathrm{ps}$ & & $0.642 \pm 0.013$ & & $0.04152 \pm 0.00056$ & & \\
\hline
\end{tabular}

Table 2 The Wilson coefficients at the scale $\mu_{b}=4.2 \mathrm{GeV}$, to NNLL accuracy in the SM

\begin{tabular}{llllllllll}
\hline$C_{1}$ & $C_{2}$ & $C_{3}$ & $C_{4}$ & $C_{5}$ & $C_{6}$ & $C_{7}$ & $C_{8}$ & $C_{9}$ & $C_{10}$ \\
\hline-0.294 & 1.017 & -0.0059 & -0.087 & 0.0004 & 0.0011 & -0.324 & -0.176 & 4.114 & -4.193 \\
\hline
\end{tabular}

\subsection{Results within the SM}

For the short-distance Wilson coefficients at the low scale $\mu_{b}=4.2 \mathrm{GeV}$, we use the numerical values collected in Table 2, which are obtained at the next-to-next-to-leading logarithmic (NNLL) accuracy within the SM [3,91,100103].

We show in Fig. 2 the SM predictions for the differential branching fraction and angular observables as a function of the dimuon invariant mass squared $q^{2}$, where the central values are plotted as red solid curves and the theoretical uncertainties, which are caused mainly by the $\Lambda_{b} \rightarrow \Lambda$ transform form factors, are labelled by the red bands. The latest experimental data from $\mathrm{LHCb}$ [97], where available, are also included in the figure for comparison. ${ }^{3}$ The SM predictions for the corresponding binned observables are presented in Table 3 .

As can be seen from Fig. 2 and Table 3, in the bin $[15,20] \mathrm{GeV}^{2}$ where both the experimental data and the lattice QCD predictions for the $\Lambda_{b} \rightarrow \Lambda$ transition form factors are most precise, the measured differential branching fraction [97], $(1.20 \pm 0.27) \times 10^{-7} \mathrm{GeV}^{-2}$, exceeds the $\mathrm{SM}$ prediction, $(0.766 \pm 0.069) \times 10^{-7} \mathrm{GeV}^{-2}$, by about $1.6 \sigma$. Although being not yet statistically significant, it is interesting to note that the deviation is in the opposite direction to what has been observed in the $B \rightarrow K^{(*)} \mu^{+} \mu^{-}[12-14]$ and $B_{S} \rightarrow \phi \mu^{+} \mu^{-}$[15-17] decays, where the measured differ-

\footnotetext{
${ }^{3}$ For the differential branching fraction, the error bars are shown both including and excluding the uncertainty from the normalization mode $\Lambda_{b} \rightarrow J / \psi \Lambda$ [98].
}

ential branching fractions favor, on the other hand, smaller values than their respective SM predictions. Also in this bin, the lepton-side forward-backward asymmetry measured by $\mathrm{LHCb}$ [97], $-0.05 \pm 0.09$, is found to be about $3.3 \sigma$ higher than the $\mathrm{SM}$ value, $-0.349 \pm 0.013$. As detailed in Ref. [81], combining the current data for $\Lambda_{b} \rightarrow \Lambda\left(\rightarrow p \pi^{-}\right) \mu^{+} \mu^{-}$ decay with that for the branching ratios of $B_{s} \rightarrow \mu^{+} \mu^{-}$and inclusive $b \rightarrow s \ell^{+} \ell^{-}$decays, Meinel and Dyk found that their fits prefer a positive shift to the Wilson coefficient $C_{9}$, which is opposite in sign compared to that found in the latest global fits of only mesonic decays [22,26,27]. This means that a simple shift in $C_{9}$ alone could not explain all the current data. Especially, a negative shift in $C_{9}$, as found in global fits of only mesonic observables, would further lower the predicted $\Lambda_{b} \rightarrow \Lambda \mu^{+} \mu^{-}$differential branching fraction.

Our SM predictions for the zero-crossing points of angular observables $A_{\mathrm{FB}}^{\ell}, A_{\mathrm{FB}}^{\ell \Lambda}$ and $Y_{2}$ read, respectively,

$$
\begin{aligned}
& \left.q_{0}^{2}\left(A_{\mathrm{FB}}^{\ell}\right)\right|_{\mathrm{SM}}=(3.95 \pm 0.62) \mathrm{GeV}^{2} \\
& \left.q_{0}^{2}\left(A_{\mathrm{FB}}^{\ell \Lambda}\right)\right|_{\mathrm{SM}}=(3.89 \pm 0.63) \mathrm{GeV}^{2} \\
& \left.q_{0}^{2}\left(Y_{2}\right)\right|_{\mathrm{SM}}=(0.35 \pm 0.10) \mathrm{GeV}^{2}
\end{aligned}
$$

The zero-crossing points of the other observables $Y_{i}(i=$ $3 \mathrm{~s}, 3 \mathrm{sc}, 4 \mathrm{~s}, 4 \mathrm{sc})$, which correspond to the case when the relative angular momentum between the $p \pi^{-}$system and the dimuon system is $(l, m)=(1, \pm 1)$, are plagued by large theoretical uncertainties. The observables $Y_{3 \mathrm{~s}}$ and $Y_{3 \mathrm{sc}}$ are predicted to be very small within SM and are, therefore, potentially good probes of NP beyond the SM [40]. 

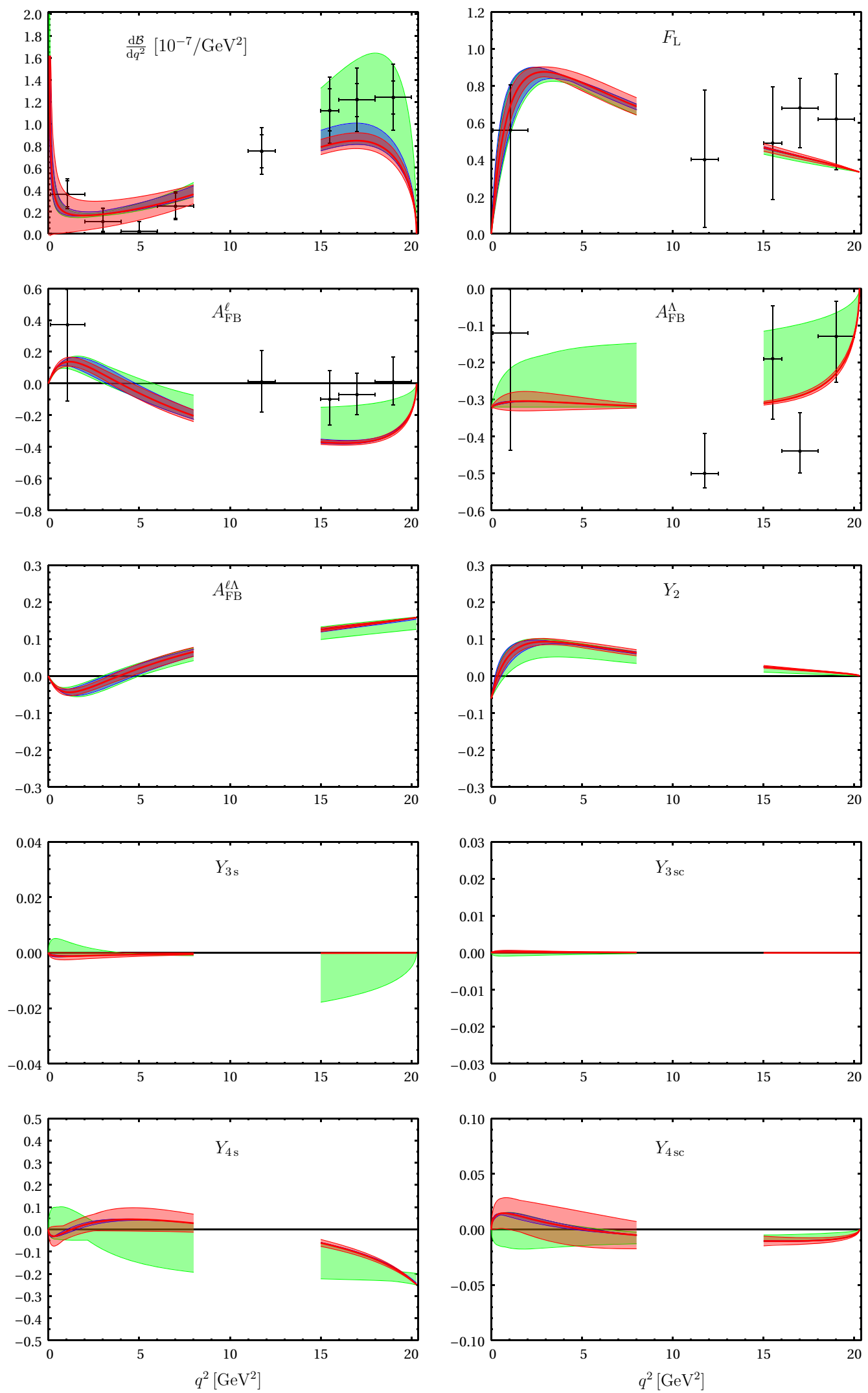

Fig. 2 The $\Lambda_{b} \rightarrow \Lambda\left(\rightarrow p \pi^{-}\right) \mu^{+} \mu^{-}$observables as a function of the dimuon invariant mass squared $q^{2}$, predicted both within the SM (central values: red solid curves, theoretical uncertainties: red bands)

and in the A2HDM (case A: blue bands and case B: green bands). The corresponding experimental data from LHCb [97], where available, are represented by the error bars 
Table 3 SM predictions for the binned differential branching fraction (in units of $10^{-7} \mathrm{GeV}^{-2}$ ) and angular observables. The first column specifies the bin ranges $\left[q_{\min }^{2}, q_{\max }^{2}\right]$ in units of $\mathrm{GeV}^{2}$

\begin{tabular}{lcccrr}
\hline & $\left\langle\mathrm{d} \mathcal{B} / \mathrm{d} q^{2}\right\rangle$ & $\left\langle F_{\mathrm{L}}\right\rangle$ & \multicolumn{1}{c}{$\left\langle A_{\mathrm{FB}}^{\ell}\right\rangle$} & \multicolumn{1}{c}{$\left\langle A_{\mathrm{FB}}^{\Lambda}\right\rangle$} & \multicolumn{1}{c}{$\left\langle A_{\mathrm{FB}}^{\ell \Lambda}\right\rangle$} \\
\hline$[0.1,2]$ & $0.24(23)$ & $0.531(92)$ & $0.104(17)$ & $-0.310(18)$ & $-0.0332(56)$ \\
{$[2,4]$} & $0.18(12)$ & $0.867(28)$ & $0.056(31)$ & $-0.306(24)$ & $-0.017(10)$ \\
{$[4,6]$} & $0.23(11)$ & $0.813(43)$ & $-0.063(39)$ & $-0.311(16)$ & $0.021(13)$ \\
{$[6,8]$} & $0.310(94)$ & $0.728(48)$ & $-0.163(39)$ & $-0.3160(88)$ & $0.053(13)$ \\
{$[1.1,6]$} & $0.20(12)$ & $0.829(33)$ & $0.011(31)$ & $-0.309(20)$ & $-0.0026(99)$ \\
{$[15,16]$} & $0.810(70)$ & $0.454(20)$ & $-0.373(14)$ & $-0.3069(55)$ & $0.1283(48)$ \\
{$[16,18]$} & $0.839(72)$ & $0.418(15)$ & $-0.371(13)$ & $-0.2890(69)$ & $0.1372(37)$ \\
{$[18,20]$} & $0.671(65)$ & $0.3711(78)$ & $-0.308(15)$ & $-0.2266(91)$ & $0.1487(19)$ \\
{$[15,20]$} & $0.766(69)$ & $0.409(13)$ & $-0.349(13)$ & $-0.2709(73)$ & $0.1394(32)$ \\
\hline & $\left\langle Y_{2}\right\rangle$ & $\left\langle Y_{3 \mathrm{~s}}\right\rangle \times 10^{-2}$ & $\left\langle Y_{3 \mathrm{sc}}\right\rangle \times 10^{-3}$ & $\left\langle Y_{4 \mathrm{~s}}\right\rangle$ & $\left\langle Y_{4 \mathrm{sc}}\right\rangle$ \\
\hline$[0.1,2]$ & $0.032(17)$ & $-0.11(12)$ & $0.25(33)$ & $-0.013(29)$ & $0.013(12)$ \\
{$[2,4]$} & $0.0918(84)$ & $-0.093(92)$ & $0.22(24)$ & $0.036(43)$ & $0.006(16)$ \\
{$[4,6]$} & $0.0838(76)$ & $-0.066(62)$ & $0.15(14)$ & $0.044(52)$ & $0.000(16)$ \\
{$[6,8]$} & $0.0696(85)$ & $-0.048(44)$ & $0.100(98)$ & $0.035(45)$ & $-0.004(14)$ \\
{$[1.1,6]$} & $0.0857(78)$ & $-0.084(84)$ & $0.19(21)$ & $0.036(42)$ & $0.004(15)$ \\
{$[15,16]$} & $0.0231(33)$ & $-0.012(12)$ & $-0.022(40)$ & $-0.070(15)$ & $-0.0105(40)$ \\
{$[16,18]$} & $0.0171(24)$ & $-0.010(12)$ & $-0.011(24)$ & $-0.106(12)$ & $-0.0104(30)$ \\
{$[18,20]$} & $0.0094(16)$ & $-0.005(10)$ & $-0.003(9)$ & $-0.1713(80)$ & $-0.0086(20)$ \\
{$[15,20]$} & $0.0157(22)$ & $-0.009(11)$ & $-0.010(22)$ & $-0.121(11)$ & $-0.0098(27)$ \\
\hline & & & & &
\end{tabular}

\subsection{Results in the A2HDM}

In this subsection, we shall investigate the impact of A2HDM on the $\Lambda_{b} \rightarrow \Lambda\left(\rightarrow p \pi^{-}\right) \mu^{+} \mu^{-}$observables. For simplicity, the alignment parameters $\varsigma_{u, d}$ are assumed to be real. As in our previous paper [82], we use the inclusive $B \rightarrow X_{s} \gamma$ branching fraction $[104,105]$ and the latest global fit results of $b \rightarrow s \ell \ell$ data $[26,81]$ to restrict the model parameters $\varsigma_{u, d}$. Under these constraints, numerically, the chargedscalar contributions to the Wilson coefficients can be divided into the following two cases [82]:

Case A : $C_{7,9,10}^{\mathrm{H}^{ \pm}}$are sizeable, but $C_{9,10}^{\prime \mathrm{H}^{ \pm}} \simeq 0$;

Case B : $C_{7}^{\mathrm{H}^{ \pm}}$and $C_{9,10}^{\prime \mathrm{H}^{ \pm}}$are sizeable, but $C_{9,10}^{\mathrm{H}^{ \pm}} \simeq 0$.

They are associated to the (large $\left|\varsigma_{u}\right|$, small $\left|\varsigma_{d}\right|$ ) and (small $\left|\varsigma_{u}\right|$, large $\left.\left|\varsigma_{d}\right|\right)$ regions, respectively; see Ref. [82] for more details. This means that the charged-scalar exchanges contribute mainly to left- and right-handed semileptonic operators in case $\mathrm{A}$ and case $\mathrm{B}$, respectively. The influences of these two cases on the $\Lambda_{b} \rightarrow \Lambda\left(\rightarrow p \pi^{-}\right) \mu^{+} \mu^{-}$observables are shown in Fig. 2, where the blue (in case A) and red (in case B) bands are obtained by varying randomly the model parameters within the ranges allowed by the global fits $[26,81,82]$, with all the other input parameters taken at their respective central values.

In case $\mathrm{A}$, the impact of $\mathrm{A} 2 \mathrm{HDM}$ is found to be negligibly small on the hadron-side forward-backward asymmetry $A_{\mathrm{FB}}^{\Lambda}$ and the observables $Y_{i}(i=3 \mathrm{~s}, 3 \mathrm{sc}, 4 \mathrm{~s}, 4 \mathrm{sc})$. For the differential branching fraction, on the other hand, visible enhancements are observed relative to the SM prediction, especially in the high $q^{2}$ region. For the remaining observables, the A2HDM only affects them in the low $q^{2}$ region, but the effect is diluted by the SM uncertainty. In order to see clearly the A2HDM effect in case A, we give in Table 4 the values of the binned observables in the bin $[15,20] \mathrm{GeV}^{2}$, including also the SM predictions, the A2HDM effect in case B, as well as the LHCb data (where available) for comparison. Although being improved a little bit, the deviations between the $\mathrm{LHCb}$ data and the theoretical values for the differential branching fraction and the lepton-side forward-backward asymmetry are still at $1.3 \sigma$ and $3.2 \sigma$, respectively. Including the A2HDM in case A, there are only small changes on the zerocrossing points:

$\left.q_{0}^{2}\left(A_{\mathrm{FB}}^{\ell}\right)\right|_{\text {case } \mathrm{A}}=(4.02 \pm 1.01) \mathrm{GeV}^{2}$,

$\left.q_{0}^{2}\left(A_{\mathrm{FB}}^{\ell \Lambda}\right)\right|_{\text {case } \mathrm{A}}=(3.96 \pm 1.02) \mathrm{GeV}^{2}$,

$\left.q_{0}^{2}\left(Y_{2}\right)\right|_{\text {case A }}=(0.37 \pm 0.20) \mathrm{GeV}^{2}$.

In case $\mathrm{B}$, however, the A2HDM has a significant influence on almost all the observables, as shown in Fig. 2. The most prominent observation is that it can enhance both the differential branching fraction and the lepton-side forward-backward asymmetry in the bin $[15,20] \mathrm{GeV}^{2}$, being now compatible with the experimental measurements at $0.2 \sigma$ and $1.3 \sigma$, 
Table 4 Comparison of our results for the

$\Lambda_{b} \rightarrow \Lambda\left(\rightarrow p \pi^{-}\right) \mu^{+} \mu^{-}$

observables with the LHCb data

(where available) in the bin

$[15,20] \mathrm{GeV}^{2}$. The

uncertainties of A2HDM results

mainly come from the $\Lambda_{b} \rightarrow \Lambda$

transition form factors and the

model parameters. The

differential branching fraction is

given in units of $10^{-7} \mathrm{GeV}^{-2}$

\begin{tabular}{llllll}
\hline & $\left\langle\mathrm{d} \mathcal{B} / \mathrm{d} q^{2}\right\rangle$ & $\left\langle F_{\mathrm{L}}\right\rangle$ & $\left\langle A_{\mathrm{FB}}^{\ell}\right\rangle$ & $\left\langle A_{\mathrm{FB}}^{\Lambda}\right\rangle$ & $\left\langle A_{\mathrm{FB}}^{\ell \Lambda}\right\rangle$ \\
\hline SM & $0.766(69)$ & $0.409(13)$ & $-0.349(13)$ & $-0.2709(73)$ & $0.1394(32)$ \\
Case A & $0.82(11)$ & $0.409(13)$ & $-0.344(16)$ & $-0.2709(73)$ & $0.1374(47)$ \\
Case B & $1.11(38)$ & $0.396(20)$ & $-0.24(12)$ & $-0.179(92)$ & $0.129(17)$ \\
LHCb [97] & $1.20(27)$ & $0.61_{-0.14}^{+0.11}$ & $-0.05(9)$ & $-0.29(8)$ & - \\
\hline & $\left\langle Y_{2}\right\rangle$ & $\left\langle Y_{3 \mathrm{~s}}\right\rangle \times 10^{-2}$ & $\left\langle Y_{3 \mathrm{sc}}\right\rangle \times 10^{-3}$ & $\left\langle Y_{4 \mathrm{~s}}\right\rangle$ & $\left\langle Y_{4 \mathrm{sc}}\right\rangle$ \\
\hline SM & $0.0157(22)$ & $-0.009(11)$ & $-0.010(22)$ & $-0.121(11)$ & $-0.0098(27)$ \\
Case A & $0.0156(23)$ & $-0.008(11)$ & $-0.011(23)$ & $-0.120(11)$ & $-0.0097(27)$ \\
Case B & $0.0110(56)$ & $-0.68(68)$ & $-0.035(39)$ & $-0.174(55)$ & $-0.007(4)$ \\
\hline
\end{tabular}

respectively (see also Table 4). The magnitude of the hadronside forward-backward asymmetry tends to become smaller in the whole $q^{2}$ region in this case, but is still in agreement with the LHCb data, with the large experimental and theoretical uncertainties taken into account. In the high (whole) $q^{2}$ region, a large effect is also observed on the asymmetry observable $Y_{3 \mathrm{~s}}\left(Y_{4 \mathrm{~s}}\right)$. Adding up the A2HDM effect in case B, the zero-crossing points are now changed to

$$
\begin{aligned}
& \left.q_{0}^{2}\left(A_{\mathrm{FB}}^{\ell}\right)\right|_{\text {case } \mathrm{B}}=(4.38 \pm 1.44) \mathrm{GeV}^{2}, \\
& \left.q_{0}^{2}\left(A_{\mathrm{FB}}^{\ell \Lambda}\right)\right|_{\text {case } \mathrm{B}}=(4.00 \pm 1.17) \mathrm{GeV}^{2}, \\
& \left.q_{0}^{2}\left(Y_{2}\right)\right|_{\text {case } \mathrm{B}}=(0.52 \pm 0.29) \mathrm{GeV}^{2},
\end{aligned}
$$

which are all significantly enhanced compared to the SM predictions (see Eq. (4.1)) and the results in case A (see Eq. (4.2)). It should be noticed that our predictions for the zero-crossing points given by Eqs. (4.1)-(4.3) are most severely affected by the hitherto unquantified systematic uncertainty coming from the non-factorizable spectatorscattering contributions at large hadronic recoil, a caveat emphasized already in Sect. 3.1.

Combining the above observations with our previous studies - the angular observable $P_{5}^{\prime}$ in $B^{0} \rightarrow K^{* 0} \mu^{+} \mu^{-}$decay could be increased significantly to be consistent with the experimental data in case B [82], we could, therefore, conclude that the A2HDM in case B is a promising alternative to the observed anomalies in $b$-hadron decays.

\section{Conclusions}

In this paper, we have investigated the A2HDM effect on the rare baryonic $\Lambda_{b} \rightarrow \Lambda\left(\rightarrow p \pi^{-}\right) \mu^{+} \mu^{-}$decay, which is mediated by the same quark-level $b \rightarrow s \mu^{+} \mu^{-}$transition as in the mesonic $B \rightarrow K^{(*)} \mu^{+} \mu^{-}$decays. In order to extract all the ten angular coefficients, we have considered the differential branching fraction $\mathrm{d} \mathcal{B} / \mathrm{d} q^{2}$, the longitudinal polarization fraction $F_{\mathrm{L}}$, the lepton-, hadron- and combined lepton-hadron-side forward-backward asymmetries $A_{\mathrm{FB}}^{\ell}, A_{\mathrm{FB}}^{\Lambda}$ and $A_{\mathrm{FB}}^{\ell \Lambda}$, as well as the other five asym- metry observables $Y_{i}(i=2,3 \mathrm{~s}, 3 \mathrm{sc}, 4 \mathrm{~s}, 4 \mathrm{sc})$. For the $\Lambda_{b} \rightarrow \Lambda$ transition form factors, we used the most recent high-precision lattice QCD calculations with $2+1$ dynamical flavours.

Taking into account constraints on the model parameters $\varsigma_{u, d}$ from the inclusive $B \rightarrow X_{s} \gamma$ branching fraction and the latest global fit results of $b \rightarrow$ sll data, we found numerically that the charged-scalar exchanges contribute either mainly to the left- or to the right-handed semileptonic operators, labelled case A and case B, respectively. The influences of these two cases on the $\Lambda_{b} \rightarrow \Lambda\left(\rightarrow p \pi^{-}\right) \mu^{+} \mu^{-}$ observables are then investigated in detail. While there are no significant differences between the SM predictions and the results in case $\mathrm{A}$, the $\mathrm{A} 2 \mathrm{HDM}$ in case $\mathrm{B}$ is much favored by the current data. Especially in the bin $[15,20] \mathrm{GeV}^{2}$ where both the experimental data and the lattice QCD predictions are most precise, the deviations between the SM predictions and the experimental data for the differential branching fraction and the lepton-side forward-backward asymmetry could be reconciled to a large extend. Also in our previous paper [82], we have found that the angular observable $P_{5}^{\prime}$ in $B^{0} \rightarrow K^{* 0} \mu^{+} \mu^{-}$decay could be increased significantly to be consistent with the experimental data in case B. Therefore, we conclude that the A2HDM in case B is a very promising solution to the currently observed anomalies in $b$-hadron decays.

Finally, it should be pointed out that more precise experimental measurements of the full angular observables, especially with a finer binning, as well as a systematic analysis of non-factorizable spectator-scattering effects in $\Lambda_{b} \rightarrow \Lambda(\rightarrow$ $\left.p \pi^{-}\right) \mu^{+} \mu^{-}$decay, would be very helpful to further deepen our understanding of the quark-level $b \rightarrow s \mu^{+} \mu^{-}$transition.

Acknowledgements The work is supported by the National Natural Science Foundation of China (NSFC) under contract Nos. 11675061, 11435003,11225523 and 11521064 . QH is supported by the Excellent Doctorial Dissertation Cultivation Grant from CCNU, under contract number 2013YBZD19.

Open Access This article is distributed under the terms of the Creative Commons Attribution 4.0 International License (http://creativecomm ons.org/licenses/by/4.0/), which permits unrestricted use, distribution, 
and reproduction in any medium, provided you give appropriate credit to the original author(s) and the source, provide a link to the Creative Commons license, and indicate if changes were made.

Funded by SCOAP ${ }^{3}$.

\section{A Wilson coefficients in A2HDM}

The coefficients $C_{i, \mathrm{XY}}^{(\prime)}(i=7,9,10$ and $\mathrm{X}, \mathrm{Y}=\mathrm{u}, \mathrm{d})$ appearing in the Wilson coefficients $C_{7,9,10}^{(\prime) \mathrm{H}^{ \pm}}$are given, respectively, as [82]

$$
\left.\begin{array}{rl}
C_{7, \mathrm{uu}}= & -\frac{1}{6} F_{6}\left(y_{t}\right), \\
C_{7, \mathrm{ud}}= & -\frac{4}{3} F_{1}\left(y_{t}\right)-\frac{80}{17} F_{2}\left(y_{t}\right)-\frac{3}{17} F_{5}\left(y_{t}\right)+\frac{1}{17} F_{6}\left(y_{t}\right), \\
C_{9, \mathrm{uu}}= & \frac{8}{9} F_{1}\left(y_{t}\right)-\frac{896}{51} F_{2}\left(y_{t}\right)-\frac{1}{17} F_{5}\left(y_{t}\right)-\frac{14}{153} F_{6}\left(y_{t}\right) \\
& -\frac{x_{t}}{2}\left(-4+\frac{1}{\sin ^{2} \theta_{W}}\right) F_{1}\left(y_{t}\right), \\
C_{10, \mathrm{uu}}= & \frac{x_{t}}{2 \sin ^{2} \theta_{W}} F_{1}\left(y_{t}\right), \\
C_{9, \mathrm{uu}}^{\prime}= & \frac{y_{t}}{x_{t}} F_{8}\left(y_{t}\right), \\
C_{9, \mathrm{ud}}^{\prime}= & \frac{y_{t}}{x_{t}} F_{7}\left(y_{t}\right), \\
C_{9, \mathrm{dd}}^{\prime}= & \frac{y_{t}}{x_{t}}\left[\frac{2}{9} F_{0}\left(x_{t}\right)+\frac{20}{9} F_{1}\left(y_{t}\right)+\frac{928}{51} F_{2}\left(y_{t}\right)\right. \\
& \left.-\frac{2}{17} F_{5}\left(y_{t}\right)-\frac{11}{153} F_{6}\left(y_{t}\right)\right], \\
C_{10, \mathrm{ud}}^{\prime}= & \frac{1}{\sin ^{2} \theta_{W}}\left[-\frac{1}{12} F_{1}\left(y_{t}\right)+\frac{30}{17} F_{2}\left(y_{t}\right)+\frac{9}{136} F_{5}\left(y_{t}\right)\right]-\frac{1}{6}\left(-4+\frac{1}{\sin ^{2} \theta_{W}}\right) F_{1}\left(y_{t}\right), \\
C_{10, \mathrm{uu}}^{\prime}= & -\frac{1}{17}\left(80 F_{2}\left(y_{t}\right)+3 F_{5}\left(y_{t}\right)-F_{6}\left(y_{t}\right)\right), \\
& (\mathrm{A} .8)
\end{array}\right)
$$

$$
\begin{aligned}
C_{10, \mathrm{dd}}^{\prime}= & -\frac{1}{\sin ^{2} \theta_{W}}\left[\frac{1}{2} F_{1}\left(y_{t}\right)+F_{2}\left(y_{t}\right)\right] \\
& +\left(-4+\frac{1}{\sin ^{2} \theta_{W}}\right) F_{2}\left(y_{t}\right),
\end{aligned}
$$

where the basic functions $F_{i}(x)$ are defined, respectively, by

$$
\begin{aligned}
& F_{0}(x)=\ln x, \\
& F_{1}(x)=\frac{x}{4-4 x}+\frac{x \ln x}{4(x-1)^{2}}, \\
& F_{2}(x)=\frac{x}{96(x-1)}-\frac{x^{2} \ln x}{96(x-1)^{2}},
\end{aligned}
$$

$$
\begin{aligned}
F_{3}(x)= & \frac{x}{8}\left[\frac{x-6}{x-1}+\frac{(3 x+2) \ln x}{(x-1)^{2}}\right] \\
F_{4}(x)= & -\frac{3 x(x-3)}{32(x-1)}+\frac{x\left(x^{2}-8 x+4\right) \ln x}{16(x-1)^{2}}, \\
F_{5}(x)= & \frac{-19 x^{3}+25 x^{2}}{36(x-1)^{3}}+\frac{\left(5 x^{2}-2 x-6\right) x^{2} \ln x}{18(x-1)^{4}}, \\
F_{6}(x)= & \frac{8 x^{3}+5 x^{2}-7 x}{12(x-1)^{3}}-\frac{(3 x-2) x^{2} \ln x}{2(x-1)^{4}} \\
F_{7}(x)= & \frac{x\left(53 x^{2}+8 x-37\right)}{108(x-1)^{4}} \\
& +\frac{x\left(-3 x^{3}-9 x^{2}+6 x+2\right) \ln x}{18(x-1)^{5}}, \\
F_{8}(x)= & \frac{x\left(18 x^{4}+253 x^{3}-767 x^{2}+853 x-417\right)}{540(x-1)^{5}} \\
& -\frac{x\left(3 x^{4}-6 x^{3}+3 x^{2}+2 x-3\right) \ln x}{9(x-1)^{6}} .
\end{aligned}
$$

\section{References}

1. S.L. Glashow, J. Iliopoulos, L. Maiani, Weak interactions with lepton-hadron symmetry. Phys. Rev. D 2, 1285-1292 (1970)

2. T. Hurth, M. Nakao, Radiative and electroweak penguin decays of B mesons. Ann. Rev. Nucl. Part. Sci. 60, 645-677 (2010). arXiv:1005.1224

3. T. Blake, G. Lanfranchi, D.M. Straub, Rare B decays as tests of the standard model. Prog. Part. Nucl. Phys. 92, 50-91 (2017). arXiv: 1606.00916

4. LHCb Collaboration, R. Aaij et al., Measurement of form-factorindependent observables in the decay $B^{0} \rightarrow K^{* 0} \mu^{+} \mu^{-}$. Phys. Rev. Lett. 111, 191801 (2013). arXiv: 1308.1707

5. LHCb Collaboration, R. Aaij et al., Angular analysis of the $B^{0} \rightarrow$ $K^{* 0} \mu^{+} \mu^{-}$decay using $3 \mathrm{fb}^{-1}$ of integrated luminosity. JHEP $\mathbf{0 2}$, 104 (2016). arXiv: 1512.04442

6. Belle Collaboration, A. Abdesselam et al., Angular analysis of $B^{0} \rightarrow K^{*}(892)^{0} \ell^{+} \ell^{-}$. In: Proceedings, LHCSki 2016-a first discussion of $13 \mathrm{TeV}$ results: Obergurgl, Austria, April 10-15, 2016 (2016). arXiv: 1604.04042

7. S. Descotes-Genon, J. Matias, M. Ramon, J. Virto, Implications from clean observables for the binned analysis of $B \rightarrow K^{*} \mu^{+} \mu^{-}$ at large recoil. JHEP 01, 048 (2013). arXiv:1207.2753

8. S. Descotes-Genon, T. Hurth, J. Matias, J. Virto, Optimizing the basis of $B \rightarrow K^{*} \ell^{+} \ell^{-}$observables in the full kinematic range. JHEP 05, 137 (2013). arXiv: 1303.5794

9. LHCb Collaboration, R. Aaij et al., Test of lepton universality using $B^{+} \rightarrow K^{+} \ell^{+} \ell^{-}$decays. Phys. Rev. Lett. 113, 151601 (2014). arXiv: 1406.6482

10. G. Hiller, F. Kruger, More model independent analysis of $b \rightarrow s$ processes. Phys. Rev. D 69, 074020 (2004). arXiv:hep-ph/0310219

11. M. Bordone, G. Isidori, A. Pattori, On the standard model predictions for $R_{K}$ and $R_{K^{*}}$. Eur. Phys. J. C 76(8), 440 (2016). arXiv: 1605.07633

12. LHCb Collaboration, R. Aaij et al., Differential branching fraction and angular analysis of the decay $B^{0} \rightarrow K^{* 0} \mu^{+} \mu^{-}$. JHEP08, 131 (2013). arXiv:1304.6325

13. LHCb Collaboration, R. Aaij et al., Differential branching fractions and isospin asymmetries of $B \rightarrow K^{(*)} \mu^{+} \mu^{-}$decays. JHEP06, 133 (2014). arXiv:1403.8044 
14. HPQCD Collaboration, C. Bouchard, G.P. Lepage, C. Monahan, H. Na, J. Shigemitsu, Standard model predictions for $B \rightarrow$ $K \ell^{+} \ell^{-}$with form factors from lattice QCD. Phys. Rev. Lett. 111(16), 162002 (2013). arXiv:1306.0434. (Erratum: Phys. Rev. Lett. 112(14), 149902(2014))

15. LHCb Collaboration, R. Aaij et al., Differential branching fraction and angular analysis of the decay $B_{s}^{0} \rightarrow \phi \mu^{+} \mu^{-}$. JHEP07, 084 (2013). arXiv:1305.2168

16. LHCb Collaboration, R. Aaij et al., Angular analysis and differential branching fraction of the decay $B_{s}^{0} \rightarrow \phi \mu^{+} \mu^{-}$. JHEP 09, 179 (2015). arXiv: 1506.08777

17. R.R. Horgan, Z. Liu, S. Meinel, M. Wingate, Calculation of $B^{0} \rightarrow K^{* 0} \mu^{+} \mu^{-}$and $B_{s}^{0} \rightarrow \phi \mu^{+} \mu^{-}$observables using form factors from lattice QCD. Phys. Rev. Lett. 112, 212003 (2014). arXiv: 1310.3887

18. S. Descotes-Genon, J. Matias, J. Virto, Understanding the $B \rightarrow K^{*} \mu^{+} \mu^{-}$anomaly. Phys. Rev. D 88, 074002 (2013). arXiv: 1307.5683

19. W. Altmannshofer, D.M. Straub, New physics in $B \rightarrow K^{*} \mu \mu$ ? Eur. Phys. J. C 73, 2646 (2013). arXiv:1308.1501

20. F. Beaujean, C. Bobeth, D. van Dyk, Comprehensive Bayesian analysis of rare (semi)leptonic and radiative $B$ decays. Eur. Phys. J. C 74, 2897 (2014). arXiv:1310.2478. (Erratum: Eur. Phys. J. C 74, 3179 (2014))

21. T. Hurth, F. Mahmoudi, On the LHCb anomaly in B $\rightarrow K^{*} \ell^{+} \ell^{-}$. JHEP 04, 097 (2014). arXiv:1312.5267

22. W. Altmannshofer, D.M. Straub, New physics in $b \rightarrow s$ transitions after LHC run 1. Eur. Phys. J. C 75(8), 382 (2015). arXiv: 1411.3161

23. T. Hurth, F. Mahmoudi, S. Neshatpour, Global fits to $b \rightarrow$ sle data and signs for lepton non-universality. JHEP 12, 053 (2014). arXiv: 1410.4545

24. F. Beaujean, C. Bobeth, S. Jahn, Constraints on tensor and scalar couplings from $B \rightarrow K \bar{\mu} \mu$ and $B_{s} \rightarrow \bar{\mu} \mu$. Eur. Phys. J. C 75(9), 456 (2015). arXiv: 1508.01526

25. D. Du, A.X. El-Khadra, S. Gottlieb, A.S. Kronfeld, J. Laiho, E. Lunghi, R.S. Van de Water, R. Zhou, Phenomenology of semileptonic B-meson decays with form factors from lattice QCD. Phys. Rev. D 93(3), 034005 (2016). arXiv: 1510.02349

26. S. Descotes-Genon, L. Hofer, J. Matias, J. Virto, Global analysis of $b \rightarrow s \ell \ell$ anomalies. JHEP 06, 092 (2016). arXiv:1510.04239

27. T. Hurth, F. Mahmoudi, S. Neshatpour, On the anomalies in the latest LHCb data. Nucl. Phys. B 909, 737-777 (2016). arXiv: 1603.00865

28. A. Khodjamirian, T. Mannel, A.A. Pivovarov, Y.M. Wang, Charmloop effect in $B \rightarrow K^{(*)} \ell^{+} \ell^{-}$and $B \rightarrow K^{*} \gamma$. JHEP 09, 089 (2010). arXiv: 1006.4945

29. A. Khodjamirian, T. Mannel, Y.M. Wang, $B \rightarrow K \ell^{+} \ell^{-}$decay at large hadronic recoil. JHEP 02, 010 (2013). arXiv:1211.0234

30. S. Jäger, J. Martin Camalich, On $B \rightarrow V \ell \ell$ at small dilepton invariant mass, power corrections, and new physics. JHEP 05, 043 (2013). arXiv: 1212.2263

31. S. Jäger, J. Martin Camalich, Reassessing the discovery potential of the $B \rightarrow K^{*} \ell^{+} \ell^{-}$decays in the large-recoil region: SM challenges and BSM opportunities. Phys. Rev. D 93(1), 014028 (2016). arXiv: 1412.3183

32. M. Ciuchini, M. Fedele, E. Franco, S. Mishima, A. Paul, L. Silvestrini, M. Valli, $B \rightarrow K^{*} \ell^{+} \ell^{-}$decays at large recoil in the Standard Model: a theoretical reappraisal. JHEP 06, 116 (2016). arXiv: 1512.07157

33. J. Lyon, R. Zwicky, Resonances gone topsy turvy-the charm of QCD or new physics in $b \rightarrow s \ell^{+} \ell^{-}$? arXiv:1406.0566

34. S. Descotes-Genon, L. Hofer, J. Matias, J. Virto, On the impact of power corrections in the prediction of $B \rightarrow K^{*} \mu^{+} \mu^{-}$observables. JHEP 12, 125 (2014). arXiv:1407.8526
35. T. Mannel, S. Recksiegel, Flavor changing neutral current decays of heavy baryons: the case $\Lambda_{b} \rightarrow \Lambda \gamma$. J. Phys. G24, 979-990 (1998). arXiv:hep-ph/9701399

36. C.-H. Chen, C.Q. Geng, Baryonic rare decays of $\Lambda_{b} \rightarrow \Lambda \ell^{+} \ell^{-}$. Phys. Rev. D 64, 074001 (2001). arXiv:hep-ph/0106193

37. C.-S. Huang, H.-G. Yan, Exclusive rare decays of heavy baryons to light baryons: $\Lambda_{b} \rightarrow \Lambda \gamma$ and $\Lambda_{b} \rightarrow \Lambda \ell^{+} \ell^{-}$. Phys. Rev. D 59, 114022 (1999). arXiv:hep-ph/9811303. (Erratum: Phys. Rev. D 61, 039901(2000))

38. P. Böer, T. Feldmann, D. van Dyk, Angular analysis of the decay $\Lambda_{b} \rightarrow \Lambda(\rightarrow N \pi) \ell^{+} \ell^{-}$. JHEP 01, 155 (2015). arXiv:1410.2115

39. T. Gutsche, M.A. Ivanov, J.G. Korner, V.E. Lyubovitskij, P. Santorelli, Rare baryon decays $\Lambda_{b} \rightarrow \Lambda l^{+} l^{-}(l=e, \mu, \tau)$ and $\Lambda_{b} \rightarrow \Lambda \gamma$ : differential and total rates, lepton- and hadron-side forward-backward asymmetries. Phys. Rev. D 87, 074031 (2013). arXiv: 1301.3737

40. G. Kumar, N. Mahajan, Asymmetries and observables for $\Lambda_{b} \rightarrow$ $\Lambda \ell^{+} \ell^{-}$. arXiv: 1511.00935

41. C.D.F. Collaboration, T. Aaltonen et al., Observation of the baryonic flavor-changing neutral current decay $\Lambda_{b} \rightarrow \Lambda \mu^{+} \mu^{-}$. Phys. Rev. Lett. 107, 201802 (2011). arXiv: 1107.3753

42. LHCb Collaboration, R. Aaij et al., Measurement of the differential branching fraction of the decay $\Lambda_{b}^{0} \rightarrow \Lambda \mu^{+} \mu^{-}$. Phys. Lett. B 725, 25-35 (2013). arXiv: 1306.2577

43. LHCb Collaboration, R. Aaij et al., Measurement of $b$-hadron production fractions in $7 \mathrm{TeV}$ pp collisions. Phys. Rev. D 85, 032008 (2012). arXiv:1111.2357

44. T. Feldmann, M.W.Y. Yip, Form factors for $\Lambda_{b} \rightarrow \Lambda$ transitions in SCET. Phys. Rev. D 85, 014035 (2012). arXiv:1111.1844. (Erratum: Phys. Rev. D 86, 079901 (2012))

45. W. Wang, Factorization of heavy-to-light baryonic transitions in SCET. Phys. Lett. B 708, 119-126 (2012). arXiv:1112.0237

46. Y.-M. Wang, Y.-L. Shen, Perturbative corrections to $\Lambda_{b} \rightarrow \Lambda$ form factors from QCD light-cone sum rules. JHEP 02, 179 (2016). arXiv: 1511.09036

47. M. Beneke, T. Feldmann, D. Seidel, Systematic approach to exclusive $B \rightarrow V \ell^{+} \ell^{-}, V \gamma$ decays. Nucl. Phys. B 612, 25-58 (2001). arXiv:hep-ph/0106067

48. M. Beneke, T. Feldmann, D. Seidel, Exclusive radiative and electroweak $b \rightarrow d$ and $b \rightarrow s$ penguin decays at NLO. Eur. Phys. J. C 41, 173-188 (2005). arXiv:hep-ph/0412400

49. B. Grinstein, D. Pirjol, Exclusive rare $B \rightarrow K^{*} \ell^{+} \ell^{-}$decays at low recoil: controlling the long-distance effects. Phys. Rev. D 70, 114005 (2004). arXiv:hep-ph/0404250

50. M. Beylich, G. Buchalla, T. Feldmann, Theory of $B \rightarrow K^{(*)} \ell^{+} \ell^{-}$ decays at high $q^{2}$ : OPE and quark-hadron duality. Eur. Phys. J. C 71, 1635 (2011). arXiv:1101.5118

51. B. Grinstein, M.J. Savage, M.B. Wise, $B \rightarrow X_{s} e^{+} e^{-}$in the six quark model. Nucl. Phys. B 319, 271-290 (1989)

52. W. Altmannshofer, P. Ball, A. Bharucha, A.J. Buras, D.M. Straub, M. Wick, Symmetries and asymmetries of $B \rightarrow K^{*} \mu^{+} \mu^{-}$ decays in the standard model and beyond. JHEP 01, 019 (2009). arXiv:0811.1214

53. C.-H. Chen, C.Q. Geng, J.N. Ng, T violation in $\Lambda_{b} \rightarrow \Lambda \ell^{+} \ell^{-}$ decays with polarized $\Lambda$. Phys. Rev. D 65, 091502 (2002). arXiv:hep-ph/0202103

54. T.M. Aliev, A. Ozpineci, M. Savci, Exclusive $\Lambda_{b} \rightarrow \Lambda \ell^{+} \ell^{-}$ decay beyond standard model. Nucl. Phys. B 649, 168-188 (2003). arXiv:hep-ph/0202120

55. T.M. Aliev, A. Ozpineci, M. Savci, New physics effects in $\Lambda_{b} \rightarrow$ $\Lambda \ell^{+} \ell^{-}$decay with lepton polarizations. Phys. Rev. D 65, 115002 (2002). arXiv:hep-ph/0203045

56. T.M. Aliev, A. Ozpineci, M. Savci, Model independent analysis of $\Lambda$ baryon polarizations in $\Lambda_{b} \rightarrow \Lambda \ell^{+} \ell^{-}$decay. Phys. Rev. D 67, 035007 (2003). arXiv:hep-ph/0211447 
57. T.M. Aliev, V. Bashiry, M. Savci, Forward-backward asymmetries in $\Lambda_{b} \rightarrow \Lambda \ell^{+} \ell^{-}$decay beyond the standard model. Nucl. Phys. B 709, 115-140 (2005). arXiv:hep-ph/0407217

58. T.M. Aliev, V. Bashiry, M. Savci, Double-lepton polarization asymmetries in $\Lambda_{b} \rightarrow \Lambda \ell^{+} \ell^{-}$decay. Eur. Phys. J. C 38, 283-295 (2004). arXiv:hep-ph/0409275

59. T.M. Aliev, M. Savci, Polarization effects in exclusive semileptonic $\Lambda_{b} \rightarrow \Lambda \ell^{+} \ell^{-}$decay. JHEP 05, 001 (2006). arXiv:hep-ph/0507324

60. A.K. Giri, R. Mohanta, Effect of R-parity violation on the rare decay $\Lambda_{b} \rightarrow \Lambda \mu^{+} \mu^{-}$. J. Phys. G31, 1559-1569 (2005)

61. A.K. Giri, R. Mohanta, Study of FCNC mediated $Z$ boson effect in the semileptonic rare baryonic decays $\Lambda_{b} \rightarrow \Lambda \ell^{+} \ell^{-}$. Eur. Phys. J. C 45, 151-158 (2006). arXiv:hep-ph/0510171

62. G. Turan, The exclusive $\Lambda_{b} \rightarrow \Lambda \ell^{+} \ell^{-}$decay with the fourth generation. JHEP 05, 008 (2005)

63. G. Turan, The exclusive $\Lambda_{b} \rightarrow \Lambda \ell^{+} \ell^{-}$decay in the general two Higgs doublet model. J. Phys. G31, 525-537 (2005)

64. T.M. Aliev, M. Savci, $\Lambda_{b} \rightarrow \Lambda \ell^{+} \ell^{-}$decay in universal extra dimensions. Eur. Phys. J. C 50, 91-99 (2007). arXiv:hep-ph/0606225

65. V. Bashiry, K. Azizi, The effects of fourth generation in single lepton polarization on $\Lambda_{b} \rightarrow \Lambda \ell^{+} \ell^{-}$decay. JHEP 07, 064 (2007). arXiv:hep-ph/0702044

66. F. Zolfagharpour, V. Bashiry, Double lepton polarization in $\Lambda_{b} \rightarrow$ $\Lambda \ell^{+} \ell^{-}$decay in the standard model with fourth generations scenario. Nucl. Phys. B 796, 294-319 (2008). arXiv:0707.4337

67. Y.-M. Wang, Y. Li, C.-D. Lu, Rare decays of $\Lambda_{b} \rightarrow \Lambda+\gamma$ and $\Lambda_{b} \rightarrow \Lambda+\ell^{+} \ell^{-}$in the light-cone sum rules. Eur. Phys. J. C 59, 861-882 (2009). arXiv:0804.0648

68. M.J. Aslam, Y.-M. Wang, C.-D. Lu, Exclusive semileptonic decays of $\Lambda_{b} \rightarrow \Lambda \ell^{+} \ell^{-}$in supersymmetric theories. Phys. Rev. D 78, 114032 (2008). arXiv:0808.2113

69. Y.-M. Wang, M.J. Aslam, C.-D. Lu, Rare decays of $\Lambda_{b} \rightarrow \Lambda \gamma$ and $\Lambda_{b} \rightarrow \Lambda l^{+} l^{-}$in universal extra dimension model. Eur. Phys. J. C 59, 847-860 (2009). arXiv:0810.0609

70. T.M. Aliev, K. Azizi, M. Savci, Analysis of the $\Lambda_{b} \rightarrow \Lambda \ell^{+} \ell^{-}$ decay in QCD. Phys. Rev. D 81, 056006 (2010). arXiv:1001.0227

71. K. Azizi, N. Katirci, Investigation of the $\Lambda_{b} \rightarrow \Lambda \ell^{+} \ell^{-}$transition in universal extra dimension using form factors from full QCD. JHEP 01, 087 (2011). arXiv:1011.5647

72. T.M. Aliev, M. Savci, Lepton polarization effects in $\Lambda_{b} \rightarrow$ $\Lambda \ell^{+} \ell^{-}$decay in family non-universal $Z^{\prime}$ model. Phys. Lett. B 718, 566-572 (2012). arXiv:1202.5444

73. L.-F. Gan, Y.-L. Liu, W.-B. Chen, M.-Q. Huang, Improved lightcone QCD sum rule analysis of the rare decays $\Lambda_{b} \rightarrow \Lambda \gamma$ And $\Lambda_{b} \rightarrow \Lambda l^{+} l^{-}$. Commun. Theor. Phys. 58, 872-882 (2012). arXiv: 1212.4671

74. K. Azizi, S. Kartal, A.T. Olgun, Z. Tavukoglu, Analysis of the semileptonic $\Lambda_{b} \rightarrow \Lambda \ell^{+} \ell^{-}$transition in the topcolorassisted technicolor model. Phys. Rev. D 88(7), 075007 (2013). arXiv: 1307.3101

75. Y.-L. Wen, C.-X. Yue, J. Zhang, Rare baryonic decays $\Lambda_{b} \rightarrow$ $\mathrm{Al}^{+} \mathrm{l}^{-}$in the TTM model. Int. J. Mod. Phys. A 28, 1350075 (2013). arXiv: 1307.5320

76. Y. Liu, L.L. Liu, X.H. Guo, Study of $\Lambda_{b} \rightarrow \Lambda l^{+} l^{-}$and $\Lambda_{b} \rightarrow p l \bar{v}$ decays in the Bethe-Salpeter equation approach. arXiv: 1503.06907

77. L. Mott, W. Roberts, Lepton polarization asymmetries for FCNC decays of the $\Lambda_{b}$ baryon. Int. J. Mod. Phys. A 30(27), 1550172 (2015). arXiv: 1506.04106

78. K. Azizi, A.T. Olgun, Z. Tavukoğlu, Comparative analysis of the $\Lambda_{b} \rightarrow \Lambda \ell^{+} \ell^{-}$decay in the SM, SUSY and RS model with custodial protection. Phys. Rev. D 92(11), 115025 (2015). arXiv: 1508.03980
79. S. Sahoo, R. Mohanta, Effects of scalar leptoquark on semileptonic $\Lambda_{b}$ decays. N. J. Phys. 18, (9), 093051 (2016). arXiv:1607.04449

80. S.-W. Wang, Y.-D. Yang, Analysis of $\Lambda_{b} \rightarrow \Lambda \mu^{+} \mu^{-}$decay in scalar leptoquark model. Adv. High Energy Phys. 2016, 5796131 (2016). arXiv:1608.03662

81. S. Meinel, D. van Dyk, Using $\Lambda_{b} \rightarrow \Lambda \mu^{+} \mu^{-}$data within a Bayesian analysis of $|\Delta B|=|\Delta S|=1$ decays. Phys. Rev. D 94(1), 013007 (2016). arXiv:1603.02974

82. Q.-Y. Hu, X.-Q. Li, Y.-D. Yang, $B^{0} \rightarrow K^{* 0} \mu^{+} \mu^{-}$Decay in the aligned two-higgs-doublet model. Eur. Phys. J. C 77(3), 190 (2017). arXiv: 1612.08867

83. A. Pich, P. Tuzón, Yukawa alignment in the two-higgs-doublet model. Phys. Rev. D 80, 091702 (2009). arXiv:0908.1554

84. LHCb Collaboration, R. Aaij et al., Measurements of the $\Lambda_{b}^{0} \rightarrow$ $J / \psi \Lambda$ decay amplitudes and the $\Lambda_{b}^{0}$ polarisation in pp collisions at $\sqrt{s}=7$ TeV. Phys. Lett. B 724, 27-35 (2013). arXiv: 1302.5578

85. W. Detmold, S. Meinel, $\Lambda_{b} \rightarrow \Lambda \ell^{+} \ell^{-}$form factors, differential branching fraction, and angular observables from lattice QCD with relativistic b quarks. Phys. Rev. D 93(7), 074501 (2016). arXiv: 1602.01399

86. C. Bourrely, I. Caprini, L. Lellouch, Model-independent description of $B \rightarrow \pi l v$ decays and a determination of $\left|V_{u b}\right|$. Phys. Rev. D 79, 013008 (2009). arXiv:0807.2722. (Erratum: Phys. Rev. D 82, 099902 (2010))

87. G.C. Branco, P.M. Ferreira, L. Lavoura, M.N. Rebelo, M. Sher, J.P. Silva, Theory and phenomenology of two-Higgs-doublet models. Phys. Rept. 516, 1-102 (2012). arXiv:1106.0034

88. X.-Q. Li, J. Lu, A. Pich, $B_{s, d}^{0} \rightarrow \ell^{+} \ell^{-}$decays in the aligned twoHiggs-doublet model. JHEP 06, 022 (2014). arXiv: 1404.5865

89. N. Cabibbo, Unitary symmetry and leptonic decays. Phys. Rev. Lett. 10, 531-533 (1963)

90. M. Kobayashi, T. Maskawa, CP violation in the renormalizable theory of weak interaction. Prog. Theor. Phys. 49, 652-657 (1973)

91. C. Bobeth, M. Misiak, J. Urban, Photonic penguins at two loops and $m_{t}$ dependence of $B R\left[B \rightarrow X_{s} l^{+} l^{-}\right]$. Nucl. Phys. B 574, 291-330 (2000). arXiv:hep-ph/9910220

92. H.H. Asatryan, H.M. Asatrian, C. Greub, M. Walker, Calculation of two loop virtual corrections to $b \rightarrow s \ell^{+} \ell^{-}$in the standard model. Phys. Rev. D 65, 074004 (2002). arXiv:hep-ph/0109140

93. C. Greub, V. Pilipp, C. Schüpbach, Analytic calculation of twoloop QCD corrections to $b \rightarrow s \ell^{+} \ell^{-}$in the high $q^{2}$ region. JHEP 12, 040 (2008). arXiv:0810.4077

94. A. Ali, T. Mannel, T. Morozumi, Forward backward asymmetry of dilepton angular distribution in the decay $b \rightarrow s \ell^{+} \ell^{-}$. Phys. Lett. B 273, 505-512 (1991)

95. G. Burdman, Short distance coefficients and the vanishing of the lepton asymmetry in $B \rightarrow V \ell^{+} \ell^{-}$. Phys. Rev. D 57, 4254-4257 (1998). arXiv:hep-ph/9710550

96. A. Ali, P. Ball, L.T. Handoko, G. Hiller, A comparative study of the decays $B \rightarrow\left(K, K^{*}\right) \ell^{+} \ell^{-}$in standard model and supersymmetric theories. Phys. Rev. D 61, 074024 (2000). arXiv:hep-ph/9910221

97. LHCb Collaboration, R. Aaij et al., Differential branching fraction and angular analysis of $\Lambda_{b}^{0} \rightarrow \Lambda \mu^{+} \mu^{-}$decays. JHEP 06, 115 (2015). arXiv: 1503.07138

98. Particle Data Group Collaboration, C. Patrignani et al., Review of particle physics. Chin. Phys. C 40(10), 100001 (2016)

99. UTfit Collaboration, http://www.utfit.org/UTfit/ ResultsSummer2016SM

100. P. Gambino, M. Gorbahn, U. Haisch, Anomalous dimension matrix for radiative and rare semileptonic $\mathrm{B}$ decays up to three loops. Nucl. Phys. B 673, 238-262 (2003). arXiv:hep-ph/0306079 
101. M. Misiak, M. Steinhauser, Three loop matching of the dipole operators for $b \rightarrow s \gamma$ and $b \rightarrow s g$. Nucl. Phys. B 683, 277-305 (2004). arXiv:hep-ph/0401041

102. M. Gorbahn, U. Haisch, Effective Hamiltonian for non-leptonic $|\Delta F|=1$ decays at NNLO in QCD. Nucl. Phys. B 713, 291-332 (2005). arXiv:hep-ph/0411071

103. D.M. Straub, flavio v0.6.0. and https://flav-io.github.io
104. Heavy Flavor Averaging Group (HFAG) Collaboration, Y. Amhis et al., Averages of b-hadron, c-hadron, and $\tau$-lepton properties as of summer 2014. arXiv: 1412.7515

105. M. Misiak et al., Updated NNLO QCD predictions for the weak radiative B-meson decays. Phys. Rev. Lett. 114(22), 221801 (2015). arXiv:1503.01789 